\title{
General Psychiatry Zolpidem dependence in an adult with bipolar affective disorder and epilepsy: A case report
}

\author{
Sujita Kumar Kar, ${ }^{\circledR}$ Suyash Dwivedi
}

To cite: Kar SK, Dwivedi S. Zolpidem dependence in an adult with bipolar affective disorder and epilepsy: A case report. General Psychiatry 2019;32:e100102. doi:10.1136/ gpsych-2019-100102

Received 18 June 2019 Revised 30 July 2019 Accepted 20 August 2019
Check for updates

(C) Author(s) (or their employer(s)) 2019. Re-use permitted under CC BY-NC. No commercial re-use. See rights and permissions. Published by BMJ.

Department of Psychiatry, King George's Medical University, Lucknow, Uttar Pradesh, India

Correspondence to Dr Sujita Kumar Kar; drsujita@gmail.com

\section{ABSTRACT}

Zolpidem is a short-acting non-benzodiazepine hypnotic agent, commonly recommended for short-term treatment of insomnia. Zolpidem has less dependence potential than benzodiazepines. Patients with mental illnesses often have disturbed sleep, for which zolpidem is often prescribed. Long-term use and self-medication (in more than recommended doses) are more likely to cause dependence. We report here a case of bipolar affective disorder with epilepsy, who developed dependence to zolpidem and had severe withdrawal symptoms. The management issues are also discussed with review of the literature.

\section{INTRODUCTION}

Zolpidem is a short-acting, non-benzodiazepine hypnotic agent. ${ }^{1}$ Zolpidem is often prescribed for short-term treatment of insomnia. It helps in the induction of sleep. It is available in immediate-release and sustained (extended)-release formulations. ${ }^{2}$ The elimination half-life of zolpidem is less than 3 hours. ${ }^{3}$ Hence, the withdrawal symptoms are expected to appear after several hours, though the risk of withdrawal is less with usage of zolpidem in therapeutic dosages (up to $10 \mathrm{mg}$ /day for approximately a 1-month period). ${ }^{3}$ Rebound insomnia, next-day hangover, cognitive impairment (disturbance of memory and co-ordination) and psychomotor disturbances are seen with use of zolpidem. ${ }^{4}$ In the elderly population, the half-life of zolpidem is prolonged, so the withdrawal symptoms are expected to appear late. The sedative effect becomes greater when co-administered with medications with central nervous system depressant properties. ${ }^{5}$ We report here the case of a patient with bipolar affective disorder with epilepsy, who developed zolpidem dependence.

\section{CASE HISTORY}

A 56-year-old man was hospitalised for odd behaviour, irrelevant talk, increased physical activity and aggressive behaviour, which were episodic in nature. These episodes often lasted for several minutes (15 to $30 \mathrm{~min}$ ) and subsided after taking $10 \mathrm{mg}$ of zolpidem. He also had decreased sleep and a burning sensation in the scalp for the past 20 days. The patient was a known case of bipolar affective disorder. At the age of 18 years, he had his first episode of mania. He had multiple mood episodes until he was 38 years of age. Most of the mood episodes were manic episodes (characterised by increased talkativeness, elevated affect, increased physical activity and grandiose thoughts) and one episode clearly indicative of depression (characterised by sadness of mood, withdrawn behaviour, decreased psychomotor activity and disturbed sleep). Past treatment details of bipolar disorder were not available. For the last 15 years (approximately), he was not taking any medications for his psychiatric illness.

He was also diagnosed with epilepsy 4 years ago and received various antiepileptic medications (levetiracetam $1500 \mathrm{mg} /$ day followed by switching to valproate $1000 \mathrm{mg}$ / day). On reviewing history, family members reported that the patient was taking 7 to 10 tablets of zolpidem of $10 \mathrm{mg}$ strength per day $(10 \mathrm{mg}$ of zolpidem every 2 to 3 hours, resulting in 70 to $100 \mathrm{mg}$ /day) for the past 2 months, which was abruptly reduced by the patient's family members for the past 20 days (to a dose of $20 \mathrm{mg} /$ day). The patient was prescribed zolpidem by a general physician for sleep disturbances. The patient had delay in onset of sleep (sleep latency ranging from 1 to 2 hours) as well as decreased duration of sleep. No precipitating factor could be identified for his sleep disturbances. He had no history of similar sleep disturbance in the past, except for the disturbances of sleep during the mood episodes. The patient used to have 2 to 3 hours of sound sleep after taking zolpidem. To have longer 
duration of sleep, the patient had started self-medicating by increasing the number of tablets. Increased consumption of tablets of zolpidem was objectionable by family members, so they tried to limit the use of zolpidem. Family members reported that whenever he was not given a tablet of zolpidem or it was delayed by an hour or so, he used to become aggressive, restless and complain of a burning sensation in the scalp, which would subside within 15 to $30 \mathrm{~min}$ of intake of zolpidem. After taking zolpidem during the day time, the patient would remain calm and quiet, but would not sleep. During a 20-day period, the patient also had multiple episodes of seizure. The patient was evaluated for seizure. Neuroimaging findings revealed diffuse cerebral atrophy but the video electroencephalogram finding was within normal limits. His blood investigations revealed hyponatremia (serum sodium 127.3 $\mathrm{mEq} /$ lit). Possibilities of seizure due to hyponatremia or due to zolpidem withdrawal were considered. He was given a relatively higher dose of zolpidem (sustained-release preparations of zolpidem up to $50 \mathrm{mg}$ /day). Hyponatremia was corrected, but his seizure persisted, so opinion was taken from neurology. As the patient had hyponatremia, he was given eslicarbazapine. At the dose of valproate $1000 \mathrm{mg}$ /day and eslicarbazapine $600 \mathrm{mg}$ / day, his seizure was well controlled. During this process, it was observed that on increasing the dose of zolpidem, his odd behaviour, irrelevant talk and increased physical activity have improved significantly. His burning sensation in the scalp had also improved. The patient's family history was insignificant. There was a history of occasional alcohol and tobacco use in the patient.

During assessment, the patient was found to be conscious and oriented but giving irrelevant answers at times with increased gesturing of hands. There was no obvious evidence of cognitive deficits. On mental status examination, his affect was perplexed in the initial few days. Subsequent interviews revealed euthymic affect. No delusions or hallucinations were elicited. Quetiapine (up to $100 \mathrm{mg} /$ day) was given to control his agitation and insomnia. The patient was shifted from an immediate-release preparation to a sustained-release preparation of zolpidem with gradual dose reduction. Over a period of 6 weeks, zolpidem was stopped. He was discharged and followed regularly, with monitoring of serum electrolytes. At 6 months' follow-up, the patient was maintained well on the antiepileptic medications and quetiapine.

\section{DISCUSSION}

There are certain unique features in our patient. The patient had developed dependence to zolpidem in a short period of time (2 months). Maximum daily consumption of zolpidem was $100 \mathrm{mg} /$ day. Certain unique withdrawal features (oddities of behaviour, increased psychomotor activity, irrelevant talk and burning sensation in the scalp) were elicited on reducing the dose of zolpidem, which subsided after increasing its dose. Most of these symptoms were episodic in nature. As zolpidem is a short-acting hypnotic agent, withdrawal symptoms are expected to reappear in frequent intervals (within hours of stoppage). In our case, the patient was having withdrawal symptoms after 2 to 3 hours of last dose of zolpidem. Evidence supports the dependence potential of zolpidem. Patients withdrawaling from zolpidem often develop insomnia, anxiety and autonomic overactivity. ${ }^{6}$ Some patients may report delirium, seizure and even psychotic features as withdrawal symptoms. ${ }^{78}$ Our patient had also developed multiple episodes of seizure after reduction of the dose of zolpidem. Hyponatremia was also present during that period. Initially, the seizures were attributed to hyponatremia. However, the seizures persisted even after correction of hyponatremia, so it is quite possible that zolpidem withdrawal might have resulted in a precipitation of seizure episodes. Patient's age, pre-existing seizure disorder, sleep deprivation and underlying hyponatremia are all potential risk factors that were attributed to current seizure episodes despite compliance to antiepileptic medications in adequate doses. Though the patient is a known case of bipolar affective disorder and epilepsy, the symptoms during the current consultation seem to be better explained by zolpidem withdrawal as they were episodically present when the patient was not taking zolpidem and improved immediately after taking zolpidem.

Shifting patients with zolpidem dependence from immediate-release preparations to sustained-release preparations and gradual dose tapering may be useful in such patients. Clinicians need to be cautious in prescribing zolpidem to elderly people, particularly those with seizure disorder or severe mental disorder. The recommendation should be for the short term and may be in the 'as and when required' basis than regular daily use.

Contributors Both authors (SKK and SD) contributed equally in collecting data, literature search and developing the manuscript.

Funding The authors have not declared a specific grant for this research from any funding agency in the public, commercial or not-for-profit sectors.

Competing interests None declared.

Patient consent for publication Next of kin consent obtained.

Provenance and peer review Not commissioned; externally peer reviewed.

Open access This is an open access article distributed in accordance with the Creative Commons Attribution Non Commercial (CC BY-NC 4.0) license, which permits others to distribute, remix, adapt, build upon this work non-commercially, and license their derivative works on different terms, provided the original work is properly cited, appropriate credit is given, any changes made indicated, and the use is non-commercial. See: http://creativecommons.org/licenses/by-nc/4.0/.

\section{REFERENCES}

1. Rohrig TP, Moore CM. Zolpidem. Forens Sci Med Pathol 2005;1:81-90.

2. Monti JM, Spence DW, Buttoo K, et al. Zolpidem's use for insomnia. Asian J Psychiatr 2017;25:79-90.

3. Holm KJ, Goa KL. Zolpidem. Drugs 2000;59:865-89. 
4. MacFarlane J, Morin CM, Montplaisir J. Hypnotics in insomnia: the experience of zolpidem. Clin Ther 2014;36:1676-701.

5 Wong E, Nguyen TV. Zolpidem use in the elderly and recent safety data. J Nurse Pract 2014:10:140-1.

6. Cavallaro R, Grazia Regazzetti M, Covelli G, et al. Tolerance and withdrawal with zolpidem. Lancet 1993;342:374-5.
7. Chien $\mathrm{C}-\mathrm{C}$, Huanga $\mathrm{H}-\mathrm{T}$, Lung $\mathrm{F}-\mathrm{W}$, et al. Zolpidem withdrawal delirium, seizure, and acute psychosis: case reports and literature review. J Subst Use 2011;16:330-8.

8. Mattoo S, Ghosh A, Chakraborty K. Newer molecules in the treatment of schizophrenia: a clinical update. Indian J Pharmacol 2011;43:729.

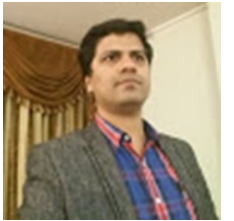

Dr.Sujita Kumar Kar is currently working as an associate professor of psychiatry in King George's Medical University, Lucknow, Uttar Pradesh, India. He is the editor in chief of Indian Journal of Health, Sexuality and Culture. He is also the editorial board member and reviewer of various national and international journals. He had written 19 book chapters and 191 articles in various national E international journals, and made 63 presentations in various national and international conferences. His research interests include brain stimulation, neuropsychiatry, psycho-sexual disorders, cognitive behaviour therapy. 